\title{
CONDIÇÕES SOCIOECONÔMICAS ESTÃO ASSOCIADAS AO OVERBITE E OVERJET? UMA AVALIAÇÃO UTILIZANDO FOTOGRAFIAS DIGITAIS
}

\section{Are socioeconomic factors associated with overbite and overjet? An evaluation using digital photographs}

\author{
Rodrigo Costa Cutrim ${ }^{(1)}$, Miyuki Damasceno Wada(2), Janailce de Almeida Lima ${ }^{(3)}$, \\ Judith Rafaelle Oliveira Pinho(4), Juliana Aires Paiva de Azevedo(5), Rafiza Felix Marão Martins ${ }^{(6)}$, \\ Cristiane Barros Leal(7), Érika Bárbara Abreu Fonseca Thomaz ${ }^{(8)}$
}

\section{RESUMO}

Objetivo: estimar a associação entre fatores socioeconômicos e más oclusões do tipo overbite e overjet em escolares em fase de dentição permanente jovem. Método: trata-se de um estudo transversal realizado em 251 escolares de sete a 15 anos, de ambos os sexos, regularmente matriculadas no ensino fundamental de escolas públicas e privadas do município de São Luís, Maranhão, Brasil. Todos os sujeitos da amostra realizaram fotografias extrabucais padronizadas de face (frontal em repouso, frontal sorrindo e perfil) e intrabucais (frontal, lateral direita e lateral esquerda em oclusão, oclusal superior e oclusal inferior), para o diagnóstico das alterações. As fotografias foram avaliadas por três examinadores em regime cego e as discordâncias foram discutidas até a obtenção de consenso. Overbite e overjet foram diagnosticados segundo critérios de Angle. Resultados: constatou-se que $35,4 \%$ das crianças apresentaram overbite e $18,3 \%$, overjet. Houve associação estatisticamente significante entre a renda familiar com overbite $(p=0.003)$ e com overjet $(p=0.029)$, observando-se que a maioria das crianças que não possuíam overbite $(69,4 \%$ ) era de famílias com baixa renda (até 2 salários mínimos). De modo semelhante, a maioria dos portadores de overjet era de famílias com renda mais elevada, ao passo que a maioria daqueles sem overjet tinha renda familiar baixa. Para as demais variáveis não houve diferenças estatisticamente significante na distribuição de frequência de overjet e overbite. Conclusão: a prevalência de overbite e overjet nos escolares estudados é alta, especialmente entre aqueles com maior renda familiar.

DESCRITORES: Má Oclusão; Prevalência; Dimensão Vertical; Dentição Mista

(1) Odontólogo; Graduado pelo Centro Universitário do Maranhão, São Luís, MA, Brasil.

(2) Odontóloga; Graduada pelo Centro Universitário do Maranhão, São Luís, MA, Brasil.

(3) Odontóloga; Graduada pelo Centro Universitário do Maranhão, São Luís, MA, Brasil.

(4) Odontóloga; Doutoranda em Saúde Coletiva pela Universidade Federal do Maranhão, São Luís, MA, Brasil; Mestre em Saúde Coletiva pela Universidade Federal do Maranhão.

(5) Odontóloga; Mestranda em Odontologia pela Universidade Federal do Maranhão, São Luís, MA, Brasil.

(6) Odontóloga; Discente do Curso de Especialização em Ortodontia - Associação Brasileira de Odontologia - Seção MA, São Luís, MA, Brasil; Mestranda em Saúde Coletiva pela Universidade Federal do Maranhão.

\section{INTRODUÇÃO}

As más oclusões (MOcl) integram um conjunto de anomalias caracterizadas pela presença de

(7) Odontóloga; Especialista em Ortodontia pela Sociedade Paulista de Ortodontia; Docente do Curso de Especialização em Ortodontia - Associação Brasileira de Odontologia - Seção MA; Mestre em Ciências da Saúde pela Universidade Federal do Maranhão;

(8) Odontóloga; Professora Adjunta do Departamento de Saúde Pública da Universidade Federal do Maranhão, São Luís, MA, Brasil; Doutora em Saúde Pública pelo Instituto de Saúde Coletiva da Universidade Federal da Bahia.

Fontes de auxílio: FAPEMA BIC-00036/08

Conflito de interesses: inexistente 
desvios no arranjo dentário e/ou na relação entre os ossos maxilomandibulares ${ }^{1}$. Têm sido evidenciadas como doenças da civilização moderna com potenciais repercussões na saúde do homem contemporâneo, podendo incluir alterações nas funções de mastigação, fonação, deglutição, dores musculares, na articulação temporomandibular, bem como problemas estéticos, e sequelas para a vida adulta nos âmbitos social, funcional e psicológico ${ }^{2,3}$. Em 2007, Suliano encontrou uma gradativa redução nas funções de fonação, deglutição e mastigação, à medida que aumentava a severidade das más-oclusões ${ }^{4}$. Há ainda indícios de que tais MOcl estão associadas com pior qualidade de vida das populações humanas ${ }^{5}$.

Dentre as principais alterações oclusais verificadas em crianças brasileiras com dentição mista destacam-se o overbite e o overjet. O overbite é definido como o trespasse entre os incisivos superiores e inferiores no plano vertical, e constitui uma característica da dentadura permanente do homem contemporâneo ${ }^{6}$. Alguns autores estabelecem padrões de normalidade para esse trespasse ${ }^{1}$, porém, quando o overbite é mais acentuado que o padrão de normalidade, passa a ser considerada como um tipo de MOcl ${ }^{1,6}$. Denomina-se overjet o trespasse horizontal em excesso da borda incisal dos dentes superiores sobre os inferiores e das cúspides vestibulares dos posteriores com relação às de seus homólogos inferiores. Tal alteração geralmente está associada a problemas de desequilíbrio da musculatura facial, decorrentes das relações dento-esqueléticas anteroposteriores ${ }^{7}$.

Em estudo sobre overjet acentuado nas crianças brasileiras, encontrou-se prevalência de $22,0 \%{ }^{8}$. Outro trabalho, realizado em 18 estados do país, com amostragem aleatória, encontrou uma prevalência de overbite de $18,09 \%$. Apesar de diferentes prevalências de $\mathrm{MOcl}$ terem sido relatadas, a maioria dos trabalhos restringe-se às regiões mais desenvolvidas do Brasil, o que dificulta a construção do perfil epidemiológico desses problemas de saúde pública no país. Além disso, foram desenvolvidos em diferentes faixas etárias e os trabalhos privilegiam critérios de classificação puramente numéricos, em detrimento das avaliações de tecidos moles, da harmonia facial e da percepção dos sujeitos ${ }^{10,11}$.

Não há consenso sobre o papel dos determinantes socioeconômicos na etiologia dos hábitos bucais deletérios e das $\mathrm{MOcl}$ dentofaciais ${ }^{3,12-16}$. Mas acredita-se que a interação de vários outros fatores pode influenciar o crescimento e o desenvolvimento dos maxilares, suscitando em MOcl. Porém, especificamente sobre a relação entre os fatores socioeconômicos e as alterações oclusais, em especial overbite e overjet ainda há dúvidas, pois poucos são os trabalhos que tratam deste assunto e os resultados são contraditórios ${ }^{1,13}$.

Alguns estudos têm demonstrado que a condição socioeconômica apresenta influência sobre a saúde, como na incidência de doenças respiratórias ${ }^{17}$, aleitamento materno ${ }^{18}$, e dificuldades de acesso aos serviços odontológicos ${ }^{19}$. Adicionalmente, alguns apontam que a condição socioeconômica está diretamente relacionada com a presença de maus hábitos bucais deletérios, o que poderia influenciar no surgimento de más oclusões ${ }^{20}$.

Deve-se considerar ainda que o custo envolvido no tratamento curativo é extremamente elevado, sendo, portanto, importante a adoção de estratégias preventivas e interceptativas, com o propósito não apenas de reduzir custos financeiros, mas também minimizar o impacto dessas alterações na saúde das populações.

Reconhecendo o overbite e o overjet como algumas das $\mathrm{MOcl}$ menos compreendidas ${ }^{2}$ e que as $\mathrm{MOcl}$ adquiriram caráter endêmico e constituem atualmente um dos principais problemas de saúde bucal, acredita-se na hipótese de que haja uma elevada prevalência destas alterações entre os adolescentes com pior situação socioeconômica na cidade avaliada. Espera-se que este trabalho seja um instrumento importante no sentido de caracterizar o perfil epidemiológico dessas alterações na população da região e, dessa forma, contribua para subsidiar a implantação de políticas públicas que visem ao controle do problema. O objetivo deste estudo é estimar a associação entre fatores socioeconômicos e MOcl do tipo overbite e overjet em escolares em fase de dentição permanente jovem.

\section{MÉTODO}

O estudo seguiu os requisitos das Resoluções 196/96 do Conselho Nacional de Saúde/Ministério da Saúde do Brasil e suas complementares. A proposta do estudo aprovada pelo Comitê de Ética em Pesquisa do Centro Universitário do Maranhão em 31/03/2008, sob no de protocolo: 00136/2008. Todos os responsáveis pelos participantes foram informados do objetivo da pesquisa e, caso aceitasse participar do estudo, eram instruídos a assinar o Termo de Consentimento Livre e Esclarecido (TCLE).

Trata-se de estudo transversal realizado em amostra composta por 251 escolares de sete a 15 anos de idade, de ambos os sexos, regularmente matriculados na $1^{\underline{a}}$ a $8^{\underline{a}}$ séries do ensino fundamental, em escolas públicas e privadas, na cidade de São Luís, Maranhão, Brasil, no período de agosto/2008 a maio/2009. 
Estima-se que na cidade de São Luís (MA) havia, na época, 998385 habitantes ${ }^{21}$, distribuídos em uma área de aproximadamente $828,0 \mathrm{~km}^{2} 22$. Segundo dados do censo escolar disponível por ocasião desta pesquisa ${ }^{23}$, estima-se que havia no município de São Luís 101.042 crianças matriculadas nas séries de $1^{\underline{a}}$ a $4^{\underline{a}}$ do ensino fundamental e 81334 nas séries de $5^{\underline{a}}$ a $8^{\underline{a}}$ do ensino fundamental, sendo que $34,6 \%$ dos estudantes estavam matriculados em escolas da rede pública estadual, $38,8 \%$ em escolas da rede pública municipal e $25,1 \%$ na rede privada de ensino.

Para a seleção dos escolares, optou-se pela técnica da amostragem probabilística por conglomerado em múltiplo estágio, com sorteio aleatório. Estimou-se, por meio do programa Epilnfo, que seria necessária uma amostra de 251 pessoas, uma prevalência de MOcl (overbite e overjet) de $17,2 \%$, e erro amostral de $4,5 \%$, considerando-se alpha de $5 \%$ e poder de $80 \%$.

A coleta dos dados foi realizada por equipe previamente treinada. As avaliações foram realizadas em uma sala na própria escola, de forma padronizada. Nos exames, utilizaram-se espelho bucal, afastadores frontal e lateral de lábio e bochecha, além de máquina fotográfica digital para a documentação fotográfica.

Foram obtidas fotografias padronizadas extrabucais (frontal em repouso, frontal sorrindo e perfil) e intrabucais (frontal, lateral direita e lateral esquerda em oclusão, oclusal superior e oclusal inferior) de todos os sujeitos da amostra. As fotografias foram realizadas em um único local em cada escola, seguindo recomendações de Siécola ${ }^{10}$, utilizando-se um aparato para iluminação do fundo com o intuito de garantir luz indireta e, dessa forma, se reduziu a presença de sombras indesejáveis nas fotografias.

O enquadramento das fotografias foi realizado de forma simplificada. Em vista frontal, a marcação horizontal da máquina fotográfica ficou paralela ao solo e a marcação vertical coincidente com a linha média da face; em vista lateral, a marcação horizontal também ficou paralela ao solo, e a marcação vertical, coincidente com o canto lateral da órbita e paralela à linha vertical ${ }^{10}$.

Para as fotografias intrabucais, padronizou-se uma determinada distância em relação aos dentes de forma que se permitiu a visualização de todos os dentes, utilizaram-se afastadores, previamente esterilizados, próprios para fotografias.

As fotografias foram avaliadas por três examinadores em regime cego e as discordâncias foram discutidas em reuniões para a obtenção de consenso.
Overbite foi definido como o trespasse entre os incisivos superiores e inferiores no plano vertical ${ }^{3}$. O overbite foi classificado como: suave: quando a borda incisal dos incisivos centrais inferiores toca o terço incisal dos incisivos centrais superiores; médio: quando os incisivos inferiores tocam o terço médio da coroa dos incisivos superiores; e, severo: quando os incisivos centrais inferiores tocam o cíngulo dos incisivos superiores ou na gengiva ${ }^{1,6}$.

Overjet foi definido como o trespasse horizontal em excesso da borda incisal dos dentes superiores sobre os inferiores e das cúspides vestibulares dos posteriores com relação ás de seus homólogos inferiores ${ }^{1}$.

Como instrumento auxiliar para a coleta dos dados, utilizou-se um questionário aplicado aos pais e aos alunos contemplando dados demográficos gerais do aluno [nome, data de nascimento para estimar a idade ("7 a 11anos" ou "12 a 15 anos"), endereço de residência, telefone, sexo ("masculino" ou "feminino"), cor/etnia (autoreferido segundo critérios do Instituto Brasileiro de Geografia e Estatística, 2006, e classificado como "branca" ou "não branca")]; dados gerais da escola [nome da escola, tipo de financiamento da escola ("pública" ou "privada")]; e caracterização socioeconômica do estudante e de sua família [escolaridade do chefe da família (categorizado em "até 8 anos" ou "9 ou mais"), renda familiar em reais ("< 2 ", "2-5" ou "> 5 salários-mínimos"), quem é o chefe da família ("pai", "mãe" ou "outro"), situação ocupacional do chefe da família ("desempregado" ou "não"), presença de banheiro intradomiciliar exclusivo para o escolar ("sim" ou "não"), série em curso, histórico de reprovação escolar do estudante ("sim" ou "não")].

As fotos foram armazenadas diariamente em arquivo de computador, protegido e com acesso limitado à equipe de pesquisadores, adotando-se o programa Adobe Photoshop para eventuais recortes das fotografias. Não foram realizadas outras alterações ou ajustes em cor, brilho, contraste ou proporções nas fotos. As imagens foram salvas em formato compactado, tomando-se o cuidado de utilizar um número sequencial de identificação dos sujeitos a fim de conexão com os demais dados dos estudantes.

Todas as informações foram armazenadas em um banco de dados criado a partir do programa Microsoft Office Excel for Windows. Em seguida, análise descritiva bivariada observou-se a distribuição de frequência das variáveis demográficas, socioeconômicas, comportamentais e biológicas em função da presença de overjet e overbite. Nessa etapa, foram identificadas possíveis diferenças nas proporções das MOcl entre as variáveis categóricas com o teste de Qui-Quadrado de Pearson $\left(\mathrm{X}^{2}\right)$, 
considerando-se o nível de significância de 5\% $(\alpha=0,05)$. O software utilizado na apuração, elaboração de tabelas, gráficos e teste de hipóteses foi o Statistica versão 7.0 desenvolvido e distribuído pela StatSof.

\section{RESULTADOS}

Neste estudo a prevalência de overbite em escolares de sete a 15 anos de idade foi de $35,4 \%$ e a de overjet, $18,3 \%$. As características sociodemográficas dos escolares são apresentadas na Tabela 1.

Houve associação estatisticamente significante entre a renda familiar e overbite $(p=0,003)$. Observou-se maior prevalência de overbite entre os estudantes cujas famílias recebiam entre 2 e 5 salários-mínimos (55,6\%). A maioria dos estudantes sem essa MOcl eram de famílias que recebiam os salários mais baixos (até 2 salários-mínimos). Para as demais variáveis não houve diferenças estatisticamente significantes na distribuição de frequência de overbite (Tabela 2).

Tabela 1 - Caracterização da amostra do estudo em 251 escolares de 7-15 anos. São Luís. Maranhão, Brasil. 2008-2009

\begin{tabular}{lccc}
\hline & $\mathbf{n}$ & $\%$ & IC 95\% \\
\hline $\begin{array}{l}\text { Sexo } \\
\text { Masculino }\end{array}$ & 113 & 45,0 & $38,9-51,2$ \\
$\quad$ Feminino & 138 & 55,0 & $48,8-61,1$ \\
Idade & & & \\
$\quad 7$ a 11 anos & 151 & 60,2 & $54,1-66,2$ \\
$\quad 12$ a 15 anos & 100 & 39,8 & $33,8-45,9$ \\
Cor & & & \\
$\quad$ Branca & 64 & 25,5 & $20,1-30,9$ \\
$\quad$ Não Branca & 187 & 74,5 & $69,1-79,9$ \\
Reprovação escolar & & & \\
$\quad$ Não & 196 & 78,1 & $73,0-83,2$ \\
$\quad$ Sim & 55 & 21,9 & $16,8-27$ \\
Renda familiar & & & \\
$\quad$ Mais de 5 SM & 27 & 10,8 & $6,9-14,6$ \\
$\quad$ Entre 2 e 5 SM & 54 & 21,5 & $16,4-26,6$ \\
$\quad$ Menor que 2 SM & 170 & 67,7 & $61,9-73,5$ \\
Chefe da família & & & \\
Pai & 121 & 48,2 & $42,0-54,4$ \\
$\quad$ Mãe & 88 & 35,1 & $29,2-41,0$ \\
$\quad$ Outro & 42 & 16,7 & $12,1-21,4$ \\
Escolaridade do chefe & & & $27,6-39,3$ \\
9 anos ou mais & 84 & 33,5 & $60,7-72,4$ \\
$\quad$ Até 8 anos & 167 & 66,5 & \\
\hline Total & $\mathbf{2 5 1}$ & & \\
\hline
\end{tabular}

$\mathrm{SM}=$ salário-mínimo em reais $(\mathrm{R} \$ 415,0)$.

IC $95 \%=$ Intervalo de Confiança a $95 \%$. 
Tabela 2 - Frequências de overbite em função de variáveis socioeconômicas e demográficas em 251 escolares de 7-15 anos. São Luís. Maranhão, Brasil. 2008-2009

\begin{tabular}{|c|c|c|c|c|c|}
\hline \multirow[b]{3}{*}{ Variáveis e categorias } & \multicolumn{4}{|c|}{ Overbite $^{*}$} & \multirow{3}{*}{$p \dagger$} \\
\hline & \multicolumn{2}{|c|}{ Sim } & \multicolumn{2}{|c|}{ Não } & \\
\hline & n (\%) & IC a $95 \%$ & n (\%) & IC a $95 \%$ & \\
\hline \multicolumn{6}{|l|}{ Sexo } \\
\hline Masculino & $45(39,8)$ & $30,8-48,8$ & $68(60,2)$ & $51,2-69,2$ & 0,185 \\
\hline Feminino & $42(30,4)$ & $22,8-38,1$ & $9(69,6)$ & $61,9-77,2$ & \\
\hline \multicolumn{6}{|l|}{ Idade } \\
\hline 7 a 11 anos & $53(35,1)$ & $27,5-42,7$ & $98(64,9)$ & $57,3-72,5$ & 0,647 \\
\hline 12 a 15 anos & $34(34,0)$ & $24,7-43,3$ & $66(66,0)$ & $56,7-75,3$ & \\
\hline \multicolumn{6}{|l|}{ Cor } \\
\hline Branca & $22(34,4)$ & $22,7-46,0$ & $42(65,6)$ & $54,0-77,3$ & 0,957 \\
\hline Não Branca & $65(34,8)$ & $27,9-41,6$ & $122(65,2)$ & $58,4-72,1$ & \\
\hline \multicolumn{6}{|l|}{ Reprovação escolar } \\
\hline Não & $71(36,2)$ & $29,5-43,0$ & $125(63,8)$ & $57,0-70,5$ & 0,604 \\
\hline Sim & $16(29,1)$ & $17,1-41,1$ & $39(70,9)$ & $58,9-82,9$ & \\
\hline \multicolumn{6}{|l|}{ Renda familiar } \\
\hline Mais de $5 \mathrm{SM}$ & $10(37,0)$ & $18,8-55,3$ & $17(63,0)$ & $44,7-81,2$ & 0,003 \\
\hline Entre 2 e $5 \mathrm{SM}$ & $30(55,6)$ & $42,3-68,8$ & $24(44,4)$ & $31,2-57,7$ & \\
\hline Menor que 2 SM & $47(27,6)$ & $20,9-34,4$ & $123(72,3)$ & $65,6-79,1$ & \\
\hline \multicolumn{6}{|l|}{ Chefe da família } \\
\hline Pai & $48(39,7)$ & $31,0-48,4$ & $73(60,3)$ & $51,6-69,0$ & 0,110 \\
\hline Mãe & $32(36,4)$ & $26,3-46,4$ & $56(63,6)$ & $53,6-73,7$ & \\
\hline Outro & $7(16,7)$ & $5,4-27,9$ & $35(83,3)$ & $72,1-94,6$ & \\
\hline \multicolumn{6}{|l|}{ Escolaridade do chefe } \\
\hline 9 anos ou mais & $36(42,9)$ & $32,3-53,4$ & $48(57,1)$ & $46,6-67,7$ & 0,050 \\
\hline Até 8 anos & $51(30,5)$ & $23,6-37,5$ & $116(69,5)$ & $62,5-76,4$ & \\
\hline
\end{tabular}

SM= salário-mínimo em reais $(\mathrm{R} \$ 415,0)$. IC 95\%= Intervalo de Confiança a 95\%.

(*) Excluídos cinco (cinco) escolares que não foram avaliados.

(†) Teste Qui-quadrado de Pearson (alfa=5\%).

A distribuição de frequência de overjet também teve diferenças estatisticamente significantes entre as categorias da renda familiar $(p=0,029)$. Dentre os portadores desta $\mathrm{MOcl}$, a maioria $(29,6 \%)$ tinha renda familiar de mais de cinco salários-mínimos, ao passo que dos estudantes sem overjet, a maioria $(84,1 \%)$ era de famílias com renda de até dois salários-mínimos. Para as demais variáveis investigadas, não houve diferenças significantes na distribuição de overjet (Tabela 3). 
Tabela 3 - Frequências de overjet em função de variáveis socioeconômicas e demográficas em 251 escolares de 7-15 anos. São Luís. Maranhão, Brasil. 2008-2009

\begin{tabular}{|c|c|c|c|c|c|}
\hline \multirow[b]{3}{*}{ Variáveis e categorias } & \multicolumn{4}{|c|}{ Overjet $^{*}$} & \multirow{3}{*}{$p \dagger$} \\
\hline & \multicolumn{2}{|c|}{ Sim } & \multicolumn{2}{|c|}{ Não } & \\
\hline & $\mathrm{n}(\%)$ & IC a $95 \%$ & $\mathrm{n}(\%)$ & IC a $95 \%$ & \\
\hline \multicolumn{6}{|l|}{ Sexo } \\
\hline Masculino & $26(23,0)$ & $15,2-30,8$ & $87(77,0)$ & $69,2-84,8$ & 0,099 \\
\hline Feminino & $19(13,8)$ & $8,0-19,5$ & $119(86,2)$ & $80,5-92,0$ & \\
\hline \multicolumn{6}{|l|}{ Idade } \\
\hline 7 a 11 anos & $25(16,6)$ & $10,6-22,5$ & $126(83,4)$ & $77,5-89,4$ & 0,496 \\
\hline 12 a 15 anos & $20(20,0)$ & $12,2-27,8$ & $80(80,0)$ & $72,2-87,8$ & \\
\hline \multicolumn{6}{|l|}{ Cor } \\
\hline Branca & $17(26,6)$ & $15,7-37,4$ & $47(73,4)$ & $62,6-84,3$ & 0,112 \\
\hline Não Branca & $28(15,0)$ & $9,9-20,1$ & $159(85,0)$ & $79,9-90,1$ & \\
\hline \multicolumn{6}{|l|}{ Reprovação escolar } \\
\hline Não & $36(18,4)$ & $12,9-23,8$ & $160(81,6)$ & $76,2-87,1$ & 0,935 \\
\hline Sim & $9(16,4)$ & $6,6-26,1$ & $46(83,6)$ & $73,9-93,4$ & \\
\hline \multicolumn{6}{|l|}{ Renda familiar } \\
\hline Mais de $5 \mathrm{SM}$ & $8(29,6)$ & $12,4-46,9$ & $19(70,4)$ & $60,3-84,2$ & 0,029 \\
\hline Entre 2 e $5 \mathrm{SM}$ & $15(27,8)$ & $15,8-39,7$ & $39(72,2)$ & $60,3-84,2$ & \\
\hline Até 2 SM & $22(12,9)$ & $7,9-18,0$ & $148(87,1)$ & $82,0-92,1$ & \\
\hline \multicolumn{6}{|l|}{ Chefe da família } \\
\hline Pai & $25(20,7)$ & $13,4-27,9$ & $96(79,3)$ & $72,1-86,6$ & 0,763 \\
\hline Mãe & $12(13,6)$ & $6,5-20,8$ & $76(86,4)$ & $79,2-93,5$ & \\
\hline Outro & $8(19,1)$ & $7,2-30,9$ & $34(80,9)$ & $69,1-92,8$ & \\
\hline \multicolumn{6}{|l|}{ Escolaridade do chefe } \\
\hline 9 anos ou mais & $21(25,0)$ & $15,7-34,3$ & $63(75,0)$ & $54,7-84,3$ & 0,040 \\
\hline Até 8 anos & $24(14,4)$ & $9,1-21,4$ & $143(85,6)$ & $80,3-92,6$ & \\
\hline
\end{tabular}

SM= salário-mínimo em reais (R\$ 415,0). IC 95\%= Intervalo de Confiança a 95\%.

(*) Excluídos cinco (cinco) escolares que não foram avaliados.

(†) Teste Qui-quadrado de Pearson. Associação estatisticamente significante entre as variáveis $(p<0.05)$.

\section{DISCUSSÃO}

$\mathrm{Na}$ amostra de 251 crianças, foi constatada uma elevada prevalência de overbite e overjet, acometendo, respectivamente $35,4 \%$ e $18,3 \%$ dos escolares. Além disso, tanto o overjet quanto overbite associaram-se de forma estatisticamente significante com a renda familiar e escolaridade do chefe da família, sendo que as maiores prevalências dessas $\mathrm{MOcl}$ ocorreram nos estudantes pertencentes aos estratos de maior renda familiar e escolaridade.

A prevalência de overbite observada este estudo $(35,4 \%)$ foi semelhante aos valores encontrados em outras investigações, as quais registraram prevalências variando de $5 \%$ a $40 \%{ }^{24-28}$. O menor valor foi registrado em estudo realizado 201 crianças e adolescentes aborígenes de 2 a 18 anos na Amazônia Peruana ${ }^{28}$. O maior foi realizado com 1024 adolescentes 13 a 15 anos em Jeddah, Arábia Saudita ${ }^{26}$. A variação encontrada pode está relacionada às diferenças de metodologia entre os estudos, especialmente o ponto de corte para classificação de overbite, tamanho e delineamento da amostra, geralmente de conveniência. No Brasil, registrou-se prevalência de $9 \%$ de overbite em 84 atletas de futebol na faixa etária de 13 a 20 anos $^{24}$. $A$ frequência de overbite mais semelhante à encontrada neste estudo foi registrada por BorzabadiFarahani et al. ${ }^{25}$, que verificaram um percentual de $39,6 \%$ de $\mathrm{MOcl}$ em 502 adolescentes de 11 a 14 anos na cidade de Isfahan, Iran.

A prevalência de overjet encontrada $(18,3 \%)$ foi muito próxima dos percentuais de $15,6 \% 7,16,2 \%^{27}$ encontrados em pesquisas semelhantes. Dados do SB Brasil apontam prevalência de $17,5 \%$ e 22,7, respectivamente, para 2003 e 2010, na população 
de adolescentes de 15 a 19 anos de idade no Brasil $^{8}$. Todavia, diferiram dos valores observados em outros estudos internacionais: $8,5 \%{ }^{28}$, $29 \%^{29}$ e $31,7 \%^{25}$. Também se pode atribuir tais resultados às diferenças metodológicas adotadas nesses estudos. Alguns analisaram exclusivamente crianças com hábitos orais deletérios, o que contribui para elevar a estimativa de frequência de MOcl dentárias. Outros utilizaram pontos de corte para definição de overjet inferiores aos adotados na presente pesquisa, a exemplo de Bhardwaj et al. ${ }^{29}$, que consideraram overjet $>2 \mathrm{~mm}$ em 622 adolescentes de 16 a 17 anos na cidade de Shimla, na Índia.

Neste estudo, overjet e overbite foram problemas mais comumente observados entre estudantes pertencentes aos estratos de maior renda familiar. No entanto, as investigações sobre a influência das condições de vida na ocorrência de problemas ortodônticos são marcadas por resultados contraditórios.

Enquanto alguns autores relatam que a necessidade de tratamento ortodôntico varia significantemente entre os diferentes estratos socioeconômicos, indicando que indivíduos que pertencem a famílias com os melhores indicadores, apresentam menores necessidades de tratamento se comparados àqueles cujas famílias possuem baixos indicadores socioeconômicos ${ }^{13,30}$, outros investigadores se opõem a esse pensamento ${ }^{31,32}$.

Nesse estudo, tanto a renda quanto a escolaridade mais elevada associaram-se ao aumento da prevalência de MOcl. Especula-se que esse fato pode estar relacionado ao maior acesso a bens de consumo, como chupetas e mamadeiras, pelas crianças cujas famílias têm maior renda. Nestas famílias, costuma-se verificar a ausência materna no domicílio, frente ao trabalho fora do lar, o que teoricamente resultaria em redução do período de amamentação ao seio, maior frequência de hábitos deletérios e, por consequência, de $\mathrm{MOcl}^{3,7,18}$. Outra pesquisa que buscou avaliar a influencia do contexto familiar nas $\mathrm{MOcl}$ não encontrou associação com fatores como renda familiar e escolaridade materna. Porém, a faixa etária das crianças e o tipo de avaliação utilizado para identificar as MOcl podem ter influenciado nos resultados. Salienta-se ainda que o tratamento ortodôntico está a cada dia mais acessível às pessoas dos estratos socioeconômicos de menor poder aquisitivo ${ }^{19,33}$, o que poderia confundir tais associações.

Outra explicação plausível para os resultados desta investigação advém da hipótese de que a perda dentária precoce, mais comumente registrada entre os mais pobres ${ }^{20}$, de certa forma, influenciaria a disposição dos dentes, aumentando o espaço livre no arco e reduzindo a ocorrência de overjet. Por outro lado, as diferenças observadas podem estar sendo confundidas por variáveis não inseridas nas análises.

Como principais limites do estudo, destacam-se o desenho seccional, que limita as inferências de associação; potenciais vieses de aferição, especialmente para variáveis socioeconômicas como renda e escolaridade, que podem causar viés por constrangimento e omissão da realidade. Outras dificuldades, especialmente na realização da técnica fotográfica de modo apropriado e padronizado e a falta de cooperação das crianças, fizeram deste estudo um desafio na busca de informações sobre a prevalência de overbite e overjet. Para tentar minimizar estes problemas, a renda e escolaridade foram coletadas em escala intervalar, esclarecendo-se ao estudante a importância e confidencialidade das informações, além do exaustivo treinamento para a aplicação dos instrumentos de coleta e realização das fotografias padronizadas, obtendo-se níveis de concordância acima de $80 \%$ para as variáveis do estudo. Outra estratégia adotada para reduzir viés de aferição foi a utilização de três avaliadores, em regime cego, para a classificação das MOcl. Por fim, a amostragem probabilística contribui para reduzir potenciais vieses de seleção ${ }^{34}$.

Ademais, a inexistência de tratamento ortodôntico nos serviços públicos do Estado do Maranhão - segundo mais pobre da federação - e a inexistência de pesquisas que caracterizem a prevalência dessas alterações oclusais em escolares de sete a 15 anos no município de São Luís, capital do Estado, tornam o estudo um instrumento importante no sentido de caracterizar o perfil epidemiológico das $\mathrm{MOcl}$ na população desta região e, dessa forma, subsidiar o planejamento de políticas públicas que visem à redução das iniquidades em saúde bucal.

\section{CONCLUSÃO}

A prevalência de overbite e overjet na dentadura mista dos escolares examinados no município de São Luís, MA, é alta, especialmente do overbite. Além disso, esses problemas ortodônticos estão relacionados a fatores socioeconômicos, sendo mais comuns entre estudantes cujas famílias têm maior renda e escolaridade. 


\section{ABSTRACT}

Purpose: to estimate the association between socioeconomic factors and occlusal changes like overbite and overjet in students aged 7 to 15 years. Method: this is a cross-sectional study in 251 students of both gender regularly enrolled in the school of public and private schools in São Luís, Maranhão, Brazil. All subjects carried out the sample photos extra-oral standardized face (front resting, smiling front and profile) and intraoral (front, right side and left side in occlusion, occlusal upper and lower occlusal) for the diagnosis of changes. The photographs were evaluated by three observers under blind and disagreements were discussed to achieve consensus. Overbite and overjet were diagnosed according to criteria of Angle. Results: it was found that $35.4 \%$ of the children were $18.3 \%$ and overbite, overjet. There was a statistically significant association between family income and overbite $(p=0.003)$ and overjet $(p=0.029)$, noting that most children who had no overbite (69.4\%) were low-income families (up to 2 minimum wage). Similarly, most people with overjet were families with higher incomes, while the majority of those without overjet had low family income. For other variables there was no statistically significant difference in frequency distribution of overjet and overbite. Conclusion: the prevalence of overbite and overjet among the studied population is high, especially among those with higher family income.

KEYWORDS: Malocclusion; Prevalence; Vertical Dimension; Dentition, Mixed

\section{REFERÊNCIAS}

1. Proffit WR, Fields HW, Sarver DM. Contemporary Orthodontics 5.ed. Canadá: Elsevier Mosby; 2013.

2. Masood Y, Masood M, Zainul NN, Araby NB, Hussain SF, Newton T. Impact of malocclusion on oral health related quality of life in young people. Health Qual Life Outcomes. 2013;11:25.

3. Tomita NE, Sheiham A, Bijella VT, Franco LJ. Relação entre determinantes socioeconômicos e hábitos bucais de risco para más-oclusões em pré-escolares. Pesqui Odontol Bras. 2000;14(2):169-75.

4. Suliano AA, Rodrigues MJ, Caldas Jr AF, Fonte PP da, Porto-Carreiro CF. Maloclusão e sua associação com alterações funcionais do sistema estomatognático entre escolares. Cad Saúde Pública. 2007;23(8):1913-23.

5. Sardenberg F, Martins MT, Bendo CB, Pordeus IA, Paiva SM, Auad SM et al. Malocclusion and oral health-related quality of life in Brazilian schoolchildren. Angle Orthod. 2013;83(1):83-9.

6. Brito HHA, Leite HR, Machado AL. Sobremordida exagerada: diagnóstico e estratégias de tratamento. Rev. Dental Press Ortodon Ortop Facial Maringá. 2009;14(3):128-57.

7. Thomaz EB, Cangussu MC, Assis AM. Maternal breastfeeding, parafunctional oral habits and malocclusion in adolescents: a multivariate analysis. Int J Pediatr Otorhinolaryngol. 2012;76(4):500-6.
8. Brasil. Ministério da Saúde. Projeto SB2010: pesquisa nacional de saúde bucal: resultados principais. Brasília, DF: Ministério da Saúde, 2011.

9. Bittencourt MAV, Machado AW. Prevalência de má oclusão em crianças entre 6 e 10 anos um panorama brasileiro. Dental Press J Orthod. 2010;15(6):113-22.

10. Siécola GS. Prevalência de padrão facial e MO em populações de duas escolas diferentes de ensino fundamental. [Dissertação]. Bauru: Faculdade de Odontologia de Bauru, Universidade de São Paulo; 2007.

11. Reis SAB, Abrão J, Capelozza Filho L, Claro CAA. Evaluation of the determinants of facial profile aesthetics. Dental Press Journal of Orthodontics. 2011;16(1):57-67.

12. Reichmuth M, Greene KA, Orsini MG, Cisneros GJ, King GJ, Kiyak HA. Occlusal perceptions of children seeking orthodontic treatment: Impact of ethnicity and socioeconomic status. Am J Orthod Dentofac Orthoped. 2005;128 (5):575-82.

13. Frazão $P$, Narvai $P C$. Socio-environmental factors associated with dental occlusion in adolescents. Am J Orthod Dentofacial Orthop. 2006;129(6):809-16.

14. Dias PF, Gleiser R. Orthodontic treatment need in a group of 9-12-year-old Brazilian schoolchildren. Braz Oral Res. 2009;23(2):182-9.

15. Marquezan M, Faraco-Junior IM, Feldens CA, Kramer PF, Ferreira SH. Association between occlusal anomalies and dental caries in 3- to 5 year-old Brazilian children. Journal of Orthodontics. 2011;38(1):8-14. 
16. Piovesan C, Antunes JLF, Guedes RS, Ardenghi TM. Impact of socioeconomic and clinical factors on child oral health-related quality of life (COHRQoL). Qual Life Res. 2010;19(9):1359-66.

17. Ma L, Liu C, Wang Y, Li S, Zhai S, Gu X et al. Mortality of neonatal respiratory failure related to socioeconomic factors in Hebei province of China. Neonatology. 2011;100(1):14-22.

18. Skafida V. Juggling work and motherhood: the impact of employment and maternity leave on breastfeeding duration: a survival analysis on Growing Up in Scotland data. Matern Child Health J. 2012;16(2):519-27.

19. Bell JF, Huebner CE, Reed SC. Oral health need and access to dental services: evidence from the National Survey of Children's Health, 2007. Matern Child Health J. 2012;16(Suppl 1):S27-34.

20. Chandrashekar BR, Reddy C, Manjunath BC, Suma S. Dental health awareness, attitude, oral health-related habits, and behaviors in relation to socio-economic factors among the municipal employees of Mysore city. Ann Trop Med Public Health. 2011;4(2):99-106.

21. Brasil. IBGE. DATASUS. Informações de Saúde. Demográficas e Socioeconômicas. [citado em 30 Nov 2012]. Disponível em: http://www2.datasus. gov.br/DATASUS/index.php?area=0206.

22. Anuário Estatístico do Maranhão. Instituto Maranhense de Estudos Socioeconômicos e Cartográficos. São Luís: IMESC; 2010.

23. Brasil. Instituto Nacional de Estudos e Pesquisas (INEP). Censo Escolar 2007. [citado em 30 Nov 2008]. Disponível em: http://www.dataescolabrasil. inep.gov.br/dataEscolaBrasil/home.seam.

24. Bernabé E, de Oliveira CM, Sheiham A. Condition-specific sociodental impacts attributed to different anterior occlusal traits in Brazilian adolescents. Eur J Oral Sci. 2007;115(6):473-8.

25. Borzabadi-Farahani A, Borzabadi-Farahani A, Eslamipour F. Orthodontic treatment needs in an urban Iranian population, an epidemiological study of 11-14 year old children. Eur J Paediatr Dent. 2009;10(2):69-74.

26. Murshid ZA, Amin HE, Al-Nowaiser AM. Distribution of certain types of occlusal anomalies among Saudi Arabian adolescents in Jeddah city. Community Dent Health. 2010;27(4):238-41.

27. Perillo L, Masucci C, Ferro F, Apicella D, Baccetti $\mathrm{T}$. Prevalence of orthodontic treatment need in southern Italian schoolchildren. Eur $\mathrm{J}$ Orthod. 2010;32(1):49-53.

28. Aliaga-Del Castillo A, Mattos-Vela MA, Aliaga-Del Castillo R, Del Castillo-Mendoza C. Malocclusions in children and adolescents from villages and native communities in the Ucayali Amazon region in Peru. Rev Peru Med Exp Salud Publica. 2011;28(1):87-91. 29. Bhardwaj VK, Veeresha KL, Sharma KR. Prevalence of malocclusion and orthodontic treatment needs among 16 and 17 year-old schoolgoing children in Shimla city, Himachal Pradesh. Indian J Dent Res. 2011;22(4):556-60.

30. Elani HW, Harper S, Allison PJ, Bedos C, Kaufman JS. Socio-economic inequalities and oral health in Canada and the United States. J Dent Res. 2012;91(9):865-70.

31. Onyeaso CO. An epidemiological survey of occlusal anomalies among secondary school children in Ibadan, Nigeria. Odontostomatol Trop. 2003;26(102):9-25.

32. Peres KG, Latorre MRDO, Sheiham A, Peres MA, Victora CG, Barros FC. Social and biological early life influences on the prevalence of open bite in Brazilian 6-year-olds. Int J Paediatr Dent. 2007 Jan;17(1):41-9.

33. Oliveira MSB de; Carneiro MC, Amorim TM, Maia VN, Alvarez AV, Vianna MIP et al. Contexto familiar, traumatismo dentário e oclusopatias em crianças em idade pré-escolar: ocorrência e fatores associados. Rev. Odontol. UNESP. 2010;39(2):81-8.

34. Rothman KJ, Greenland S, Lash TL. Epidemiologia Moderna. 3 ed. Porto Alegre: Artmed; 2011.

Recebido em: 30/11/2012

Aceito em: 27/03/2013

Endereço para correspondência:

Érika Barbara Abreu Fonseca Thomaz

Rua Barão de Itapary, 155 - Centro

São Luís - MA - Brasil

CEP: 65020-070

E-mail: ebthomaz@globo.com 